\title{
Padrões de crescimento de machos e fêmeas de tilápias do Nilo (Oreochromis niloticus) da variedade GIFT ${ }^{1}$
}

\section{Growth patterns of male and female of Nile tilapia (Oreochromis niloticus) GIFT variety}

\author{
Aline Mayra da Silva Oliveira ${ }^{2 *}$; Carlos Antonio Lopes de Oliveira ${ }^{3,4}$; \\ Bárbara Joyce Akemi Matsubara ${ }^{3}$; Sheila Nogueira de Oliveira ${ }^{3}$; \\ Natali Miwa Kunita ${ }^{3}$; Grazyella Massako Yoshida ${ }^{3}$; Ricardo Pereira Ribeiro ${ }^{3}$
}

\section{Resumo}

Objetivou-se estimar o padrão de crescimento de tilápias GIFT por meio do modelo não linear de Gompertz e verificar se existem diferenças no padrão de crescimento de machos e fêmeas. O modelo de Gompertz foi testado com oito variações, sendo o primeiro modelo sem nenhuma restrição no espaço paramétrico e os demais modelos com diferentes números de restrições para descrever o padrão de crescimento em peso corporal, comprimento total, altura do corpo, largura do corpo e tamanho da cabeça. O modelo de melhor ajuste para peso, comprimento total, largura do corpo e tamanho da cabeça foi o modelo 3 e para altura do corpo foi o modelo 1. Comparando o incremento do peso com as demais características, observou-se que os pontos de inflexão das características morfométricas variaram de 121,2 a 156,2 dias para fêmeas e 134,1 a 166,6 dias para machos, enquanto que para peso o ponto de inflexão foi de 340,4 e 322,9 dias para machos e fêmeas respectivamente, indicando que a forma dos peixes foi determinada em idade menor que o peso. O padrão de crescimento das partes constituintes do corpo e do peso de machos e fêmeas de tilápias GIFT é diferente, confirmando o dimorfismo sexual. Machos e fêmeas apresentam mesmo crescimento relativo no ponto de inflexão para a maioria das características avaliadas, com exceção da altura do corpo.

Palavras-chave: Aceleração, crescimento relativo, incremento corporal, medidas morfométricas, modelo de crescimento, tilapicultura

\begin{abstract}
The aim of this work was to estimate the growth pattern of GIFT tilapia using nonlinear model Gompertz and verifying the differences between growth pattern of males and females. The Gompertz model has been tested within eight variations. The first model had not restriction on the parametric space, but the other models presented several numbers of restrictions, ranged from 1 to 3 restrictions. Were estimated the growth pattern for body weight, total length, body height, body width and head size.
\end{abstract}

\footnotetext{
${ }^{1}$ Parte da Dissertação de Mestrado da primeira autora apresentada à Universidade Estadual de Mato Grosso do Sul, UEMS, como parte dos requisitos para obtenção do título de Mestre em Zootecnia. Bolsista CAPES.

2 Discente de Mestrado do Programa de Pós-Graduação em Zootecnia, Produção Animal no Cerrado-Pantanal, UEMS, Unidade Universitária de Aquidauana, Aquidauana, MS. E-mail: alimayrazoo@yahoo.com.br

${ }^{3}$ Grupo de Pesquisa PeixeGen, Universidade Estadual de Maringá, UEM, Centro de Ciências Agrárias, Maringá, PR. E-mail: caloliveira@uem.br; baakemi@hotmail.com; she_uem@hotmail.com; nat_kunita@hotmail.com; grazyoshida@hotmail.com; rpribeiro@uem.br

${ }^{4}$ Prof. do Programa de Pós-Graduação em Zootecnia, Produção Animal no Cerrado-Pantanal, UEMS, Unidade Universitária de Aquidauana, Aquidauana, MS. E-mail: caloliveira@uem.br

* Autor para correspondência
} 
Model three was the fittest for total length, weight, body width, and size of the head, while for height of the body the model one has the better fit than the other models. Comparing the weight increment with the other characteristics, it was observed that the inflection points of the morphometric characteristics ranged from 121.2 to 156.2 day for females and 134.1 to 166.6 day for males, while that for weight the inflection point was 340.4 and 322.9 day to males and females respectively, showing that the form of the fish was determined in smaller age than weight. The growth pattern of the body shares constituents and weight of males and females GIFT differ, confirming sexual dimorphism. Males and females presented equal growth relative in the inflection point for the characteristics majority of evaluated, except for body height.

Key words: Acceleration, relative growth, body increment, morphometric measures, growth model, tilapia culture

\section{Introdução}

O crescimento da tilapicultura no mundo e a contínua intensificação dos sistemas de cultivo resultaram na busca constante por linhagens de desempenho superior (COSTA et al., 2009), de maneira que para avaliar estas linhagens, os estudos de curvas de crescimento por meio do ajuste de equações de predição do peso em função da idade do animal tem sido de grande importância, pois condensam as informações de uma série de dados em um pequeno conjunto de parâmetros biologicamente interpretáveis (SANTOS et al., 2007b) e podem ser utilizados na identificação de animais mais precoces ou mais produtivos para serem empregados em programas de seleção e melhoramento genético (BARICHELLO et al., 2009).

A curva típica de crescimento, durante a vida, apresenta forma sigmoide, ou seja, o crescimento durante a primeira etapa da vida é lento, seguido de um período de autoaceleração, até atingir o ponto máximo da taxa de crescimento, por volta da puberdade, quando ocorre uma fase de autodesaceleração (BERG; BUTTERFIELD, 1976). A avaliação de modelos matemáticos para descrever o padrão de crescimento tem indicado que modelos de regressão não linear são os mais adequados, Mazzini et al. (2003) citam que dentre as funções não lineares mais utilizadas para ajustar as relações peso-idade destacam-se Richards, Brody, Von Bertalanffy, Logístico e Gompertz. Entretanto, o modelo de Gompertz dentre as demais funções é muito usado (COSTA et al., 2009), pois ele expressa massa em relação à idade do animal, utilizando apenas três parâmetros, diferindo-se das outras curvas por apresentar massa corporal superior à zero, ou seja, considera que o animal já nasce com alguma massa (FIALHO, 1999).

A aplicação de modelos não lineares para o ajuste de curvas de crescimento de tilápias do Nilo, de acordo com Santos et al. (2007b), foi satisfatória em todos os modelos, mas os modelos de Gompertz e Von Bertalanffy apresentaram-se mais indicados, tanto para a linhagem Chitralada quanto para a Supreme.

Para a descrição do crescimento de peixes jovens, Gamito (1998) salienta que o modelo de Gompertz pode ser mais apropriado, pois representa adequadamente o curso de crescimento de peixes observados por um curto período de tempo. Borges (2008) afirma que o modelo Gompertz tanto pode ser usado para descrever o crescimento em peso quanto em comprimento.

Com o intuito de auxiliar a compreensão do crescimento de tilápias do Nilo da variedade GIFT (Genetically Improved Farming Tilapia) objetivouse estimar o padrão de crescimento por meio do modelo de regressão não linear Gompertz e verificar se existem diferenças no padrão de crescimento de machos e fêmeas deste grupo genético.

\section{Material e Métodos}

O conjunto de dados utilizado neste estudo foi cedido pelo grupo de pesquisa PeixeGen da Universidade Estadual de Maringá - UEM e continha 
informações de peso vivo e medidas morfométricas de 972 machos e 747 fêmeas de tilápias do Nilo da variedade GIFT (Genetically Improved Farming Tilapia). Os peixes utilizados foram produzidos na Estação de Piscicultura UEM - CODAPAR, pertencente à Universidade Estadual de Maringá, no município de Floriano - PR (23 31' 8' S 52 2' 21 ” $\mathrm{O}$ ), durante a estação reprodutiva compreendida entre os meses de novembro de 2008 a fevereiro de 2009. Os peixes, após o nascimento foram mantidos com as mães em hapas de $1 \mathrm{~m}^{3}$ até o final da estação reprodutiva, em seguida foram transferidos pata tanques de alevinagem, sendo mantidos em hapas de $1 \mathrm{~m}^{3}$ em grupos de 50 indivíduos por cerca de 30 dias.

Após atingirem peso superior a 10 gramas, os animais foram identificados, utilizando-se microchips implantados na cavidade visceral. Uma vez identificados se realizou a primeira biometria e em seguida os peixes foram enviados para a unidade demonstrativa de produção em tanques rede da UEM, no Município de Diamante do Norte - PR (22 39' 24"' S 52 46' 51'” O), no Rio do Corvo, tributário do Rio Paranapanema no reservatório de Rosana.

Os pesos médios iniciais foram de 23,83 $\pm 7,7$ g e 22,15 $\pm 6,9$ g para machos e fêmeas, respectivamente. Os valores médios inicias de $\mathrm{CT}$, $\mathrm{AC}, \mathrm{LC}$ e TC foram 10,2 $\pm 1,1 \mathrm{~cm}, 9,9 \pm 1,04 \mathrm{~cm}$; $2,7 \pm 0,4 \mathrm{~cm}, 2,6 \pm 0,4 \mathrm{~cm} ; 1,4 \pm 0,2 \mathrm{~cm}, 1,1 \pm 0,3$ $\mathrm{cm} ; 2,2 \pm 0,4 \mathrm{~cm}, 2,1 \pm 0,4 \mathrm{~cm}$ dos machos e fêmeas, respectivamente.

Os peixes foram cultivados em três tanques rede com volume de $6 \mathrm{~m}^{3}$ e densidade média de 100 peixes $/ \mathrm{m}^{3}$ durante os meses de maio a setembro de 2009, realizando-se cinco biometrias em intervalos de aproximadamente 30 dias. A alimentação era fornecida as 8, 13 e 17 horas e continha 32\% PB. $\mathrm{O}$ ajuste para fornecimento da ração foi de acordo com as biometrias realizadas no decorrer do cultivo.

Os dados biométricos mensurados foram:

Peso corporal em gramas (Peso);
Comprimento total em $\mathrm{cm}$ (CT - compreendido da extremidade anterior da cabeça e o final da nadadeira caudal);

Altura do corpo em $\mathrm{cm}$ ( $\mathrm{AC}$ - medida à frente do $1^{\circ}$ raio da nadadeira dorsal);

Largura do corpo em $\mathrm{cm}$ (LC - à frente do $1^{\circ}$ raio da nadadeira dorsal);

Tamanho da cabeça em cm (TC - compreendido entre a extremidade anterior da cabeça e o bordo caudal do opérculo) (Figura 1).

Figura 1. Medidas morfométricas mensuradas em cada peixe. Autora Aline Mayra da Silva Oliveira

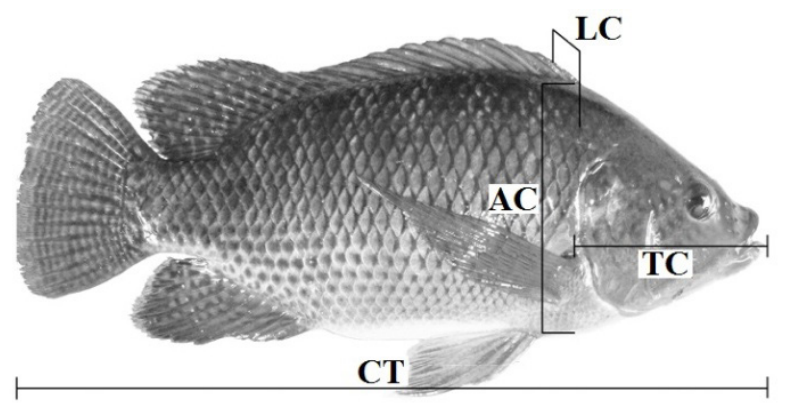

Fonte: Elaboração dos autores.

Na obtenção do peso foi utilizada uma balança digital com precisão de $0,1 \mathrm{~g}$. O comprimento total e o tamanho da cabeça foram mensurados utilizando um ictiômetro e as demais medidas foram realizadas com auxílio de um paquímetro.

Para descrever o comportamento do crescimento dos peixes foi utilizado o modelo de regressão não linear de Gompertz (FIALHO, 1999), descrito a seguir:

$$
\mathrm{M}=\mathrm{A} \cdot \mathrm{e}^{-\mathrm{e}^{-\mathrm{B} \cdot(\mathrm{t}-\mathrm{C})}}
$$

Em que:

$M=$ peso $(\mathrm{g})$ ou tamanho $(\mathrm{cm})$ estimado à idade $t$

$A=$ peso $(\mathrm{g})$ ou tamanho $(\mathrm{cm})$ assintótico quando $t$ tende a mais infinito, ou seja, este parâmetro pode 
ser interpretado como peso ou tamanho à idade adulta;

$B=$ crescimento relativo no ponto de inflexão $(\mathrm{g} /$ dia por $\mathrm{g}$ ou $\mathrm{cm} /$ dia por $\mathrm{cm}$ );

$C$ = idade no ponto de inflexão (dias);

$t=$ idade (dias);

$e=2,718281828459$.

Os modelos foram ajustados para descrever o padrão de crescimento de machos e fêmeas em peso corporal (peso), comprimento total (CT), altura do corpo (AC), largura do corpo (LC) e tamanho da cabeça (TC). Os parâmetros da curva de crescimento foram estimados pelo método de Gauss Newton modificado no procedimento NLIN do programa SAS versão 9.1.3. (SAS, 2002, 2004).

Foram testados oito modelos, o primeiro sem nenhuma restrição no espaço paramétrico (M1), considerando que os parâmetros da curva de crescimento de Gompertz para machos e fêmeas foram diferentes. Os demais modelos apresentaram diferentes números de restrições, descrevendo curvas de crescimento de machos e fêmeas, que apresentavam um, dois ou três parâmetros do modelo comuns, conforme apresentado na Tabela 1.

Tabela 1. Descrição dos modelos testados.

\begin{tabular}{|c|c|c|c|c|c|c|c|c|}
\hline \multicolumn{9}{|c|}{ Ajuste dos modelos } \\
\hline & $\mathbf{M}$ & $\mathbf{M}_{2}$ & $\mathbf{M}_{3}$ & $\mathbf{M}_{4}$ & $\mathbf{M}_{5}$ & $\mathbf{M}_{6}$ & $\mathbf{M}_{7}$ & $\mathbf{M}$ \\
\hline \multirow{9}{*}{ 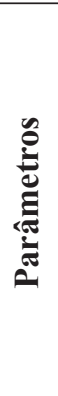 } & $a_{\mathrm{i}}$ & $a_{\mathrm{i}}$ & $a_{\mathrm{i}}$ & - & $a_{\mathrm{i}}$ & - & - & - \\
\hline & $b_{\mathrm{i}}$ & $b_{\mathrm{i}}$ & - & $b_{\mathrm{i}}$ & - & - & $b_{\mathrm{i}}$ & - \\
\hline & $c_{i}$ & - & $c_{i}$ & $c_{i}$ & - & $c_{i}$ & - & - \\
\hline & $a_{\mathrm{j}}$ & $a_{\mathrm{j}}$ & $a_{\mathrm{j}}$ & - & $a_{\mathrm{j}}$ & - & - & - \\
\hline & $b_{\mathrm{j}}$ & $b_{j}$ & - & $b_{\mathrm{j}}$ & - & - & $b_{j}$ & - \\
\hline & $c_{\mathrm{j}}$ & - & $c_{j}$ & $c_{j}$ & - & $c_{j}$ & - & - \\
\hline & - & - & - & $A$ & - & $A$ & $A$ & $A$ \\
\hline & - & - & $B$ & - & $B$ & $B$ & - & $B$ \\
\hline & - & $C$ & - & - & $C$ & - & $C$ & $C$ \\
\hline
\end{tabular}

Os parâmetros a, b e c são do modelo de Gompertz sendo $\mathrm{i}=$ machos e $\mathrm{j}=$ =ềmeas. Parâmetros descritos por letras maiúsculas são comuns para machos e fêmeas.

Fonte: Elaboração dos autores.

A adequacidade dos diferentes modelos demais, para característica peso (Tabela 2). foi avaliada por meio do teste da razão de verossimilhança com aproximação pela estatística qui-quadrado $\left(\mathrm{X}^{2}\right)$ conforme descrito por Regazzi e Silva (2004).

\section{Resultados e Discussão}

Os modelos utilizados descreveram adequadamente o padrão de crescimento das características estudadas para machos e fêmeas. $\mathrm{O}$ modelo 7 apresentou valor assintótico e idade no ponto de inflexão superestimados em relação aos

De acordo com os resultados, os modelos que apresentaram melhor ajuste foram aqueles que consideraram a existência de diferentes parâmetros para machos e fêmeas. Dessa forma, o modelo 3 foi mais adequado que o modelo mais complexo $\left(\mathrm{M}_{1}\right)$ para as variáveis peso, CT, LC e TC. Porém, para AC o modelo 1 apresentou melhor ajuste que os demais, indicando que os três parâmetros do modelo de Gompertz foram diferentes para machos e fêmeas (Tabela 2). 
Tabela 2. Estimativas dos parâmetros dos modelos sem restrição e modelos com restrições ajustados para peso, comprimento total (CT), largura do corpo (LC), altura do corpo (AC) e tamanho da cabeça (TC) de machos e fêmeas de tilápias GIFT e respectivas somas de quadrados do resíduo da regressão (SQRR).

\begin{tabular}{|c|c|c|c|c|c|c|c|c|c|}
\hline \multirow{2}{*}{ 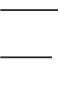 } & \multirow{2}{*}{ Parâmetros } & \multicolumn{8}{|c|}{ Estimativas dos parâmetros dos modelos } \\
\hline & & $\mathbf{M}_{1}$ & $\mathbf{M}_{2}$ & $\mathbf{M}_{3}$ & $\mathrm{M}_{4}$ & $\mathbf{M}_{5}$ & $\mathbf{M}_{6}$ & $\mathbf{M}_{7}$ & $\mathbf{M}_{8}$ \\
\hline \multirow{10}{*}{ 总 } & $a_{i}$ & 1166,7 & 1082,8 & 1111,2 & - & 1063,5 & - & - & - \\
\hline & $b_{i}^{i}$ & 0,00551 & 0,00574 & - & 0,00590 & - & - & 0,00190 & - \\
\hline & $c_{i}$ & 348,2 & & 340,4 & 328,7 & - & 494,8 & - & - \\
\hline & $a_{j}$ & 627,6 & 754,6 & 695,6 & - & 771,6 & - & - & - \\
\hline & $b_{j}^{j}$ & 0,00604 & 0,00540 & - & 0,00460 & - & - & 0,00197 & - \\
\hline & $c_{j}^{j}$ & 306,2 & - & 322,9 & 391,3 & - & 528,1 & - & - \\
\hline & $A$ & - & - & - & 1030,6 & - & 2465,0 & 31220,4 & 868,1 \\
\hline & $B$ & - & - & 0,00566 & - & 0,00566 & 0,00371 & - & 0,00584 \\
\hline & $C$ & - & 336,4 & - & - & 335,4 & - & 1101,2 & 324,5 \\
\hline & SQRR & 17459209 & 17463044 & 17460613* & 17477198 & 17522904 & 17906294 & 18130265 & 22784071 \\
\hline \multirow{10}{*}{ E } & $a_{i}$ & 40,5234 & 38,9304 & 39,6213 & - & 38,7985 & - & - & - \\
\hline & $b_{i}^{i}$ & 0,00543 & 0,00572 & - & 0,00584 & - & - & 0,00731 & - \\
\hline & $c_{i}$ & 165,1 & - & 160,9 & 154,9 & - & 171,6 & - & - \\
\hline & $a_{i}$ & 33,4721 & 36,2419 & 34,6711 & - & 35,4588 & - & - & - \\
\hline & $b$ & 0,00589 & 0,00528 & - & 0,00492 & - & - & 0,00558 & - \\
\hline & $c$ & 143,0 & - & 149,1 & 168,8 & - & 192,5 & - & - \\
\hline & $A$ & - & - & - & 38,3252 & - & 42,3121 & 33,4555 & 37,021 \\
\hline & $B$ & - & - & 0,00560 & - & 0,00563 & 0,00482 & - & 0,00567 \\
\hline & $C$ & - & 157,6 & - & - & 155,7 & - & 136,5 & 153,9 \\
\hline & SQRR & 29246,9 & 29274,8 & $29253,8^{*}$ & 29312,8 & 29398,5 & 30388,5 & 29994,8 & 34973,1 \\
\hline \multirow{10}{*}{$\underset{\psi}{U}$} & $a_{i}$ & 9,8414 & 9,4895 & 9,6463 & - & 9,5082 & - & - & - \\
\hline & $b_{i}^{1}$ & 0,00850 & 0,00903 & - & 0,00929 & - & - & 0,00998 & - \\
\hline & $c_{i . .}$ & 134,6 & - & 132,5 & 129,3 & - & 128,8 & - & - \\
\hline & $a_{j}$ & 8,1357 & 8,7859 & 8,3906 & - & 8,5393 & - & - & - \\
\hline & $b_{j}$ & 0,00946 & 0,00819 & - & 0,00724 & - & - & 0,00728 & - \\
\hline & $c^{j}$ & 121,2 & - & 123,8 & 137,0 & - & 150,1 & - & - \\
\hline & $A$ & - & - & - & 9,3745 & - & 9,8245 & 9,1234 & 9,0247 \\
\hline & $B$ & - & - & 0,00883 & - & 0,00887 & 0,00769 & - & 0,00894 \\
\hline & $C$ & - & 130,0 & - & - & 129,0 & - & 128,8 & 128,1 \\
\hline & SQRR & $4593,9 *$ & 4608,1 & 4596,9 & 4625,7 & 4615,8 & 4857,0 & 4654,2 & 5359,3 \\
\hline \multirow{10}{*}{ בَ } & $a_{i}$ & 4,3681 & 4,2827 & 4,3500 & - & 4,3033 & - & - & - \\
\hline & $b_{i}^{i}$ & 0,00869 & 0,00898 & - & 0,00924 & - & - & 0,00991 & - \\
\hline & $c_{i \ldots}$ & 134,6 & - & 134,1 & 130,9 & - & 130,7 & - & - \\
\hline & $a_{j}$ & 3,8328 & 4,0026 & 3,8594 & - & 3,9127 & - & - & - \\
\hline & $b_{j}^{j}$ & 0,00888 & 0,00822 & - & 0,00746 & - & - & 0,00751 & - \\
\hline & $c$ & 126,9 & - & 127,6 & 138,3 & - & 149,4 & - & - \\
\hline & $A$ & - & - & - & 4,2231 & - & 4,3997 & 4,1122 & 4,1131 \\
\hline & $B$ & - & - & 0,00876 & - & 0,00878 & 0,00785 & - & 0,00883 \\
\hline & $C$ & - & 132,1 & - & - & 131,5 & - & 130,4 & 130,8 \\
\hline & SQRR & 1389,8 & 1390,6 & $1389,9^{*}$ & 1392,6 & 1392,1 & 1428,4 & 1398,4 & 1510,0 \\
\hline \multirow{10}{*}{ U } & $a_{i}$ & 9,9141 & 9,4199 & 9,5692 & - & 9,3791 & - & - & - \\
\hline & $b_{i}^{i}$ & 0,00586 & 0,00624 & - & 0,00638 & - & - & 0,00805 & - \\
\hline & $c_{i}^{i}$ & 172,4 & - & 166,6 & 161,3 & - & 176,2 & - & - \\
\hline & $a_{j}$ & 7,8914 & 8,6956 & 8,3336 & - & 8,5136 & - & - & - \\
\hline & $b_{j}$ & 0,00664 & 0,00581 & - & 0,00536 & - & - & 0,00609 & - \\
\hline & $c_{j}^{j}$ & 147,5 & - & 156,2 & 175,7 & - & 196,6 & - & - \\
\hline & $A$ & - & - & - & 9,272 & - & 10,2016 & 8,442 & 8,9141 \\
\hline & $B$ & - & - & 0,00613 & - & 0,00616 & 0,00529 & - & 0,00622 \\
\hline & $C$ & - & 163,8 & - & - & 162,0 & - & 143,6 & 160,2 \\
\hline & SQRR & 2785,6 & 2788,7 & $2786,8^{*}$ & 2792,4 & 2795,7 & 2867,9 & 2850,5 & 3168,2 \\
\hline
\end{tabular}

Os parâmetros a, b e c são do modelo de Gompertz sendo i=machos e j=fêmeas. Parâmetros descritos por letras maiúsculas são comuns para machos e fêmeas. *Modelo de melhor ajuste pela estatística $\mathrm{X}^{2}(\mathrm{P}<0,05)$.

Fonte: Elaboração dos autores. 
Os resultados encontrados indicaram que para a característica peso, houve diferença no padrão de crescimento entre machos e fêmeas, uma vez que o peso assintótico e a idade no ponto de inflexão dos machos foram maiores para todos os modelos testados em relação às fêmeas. Isso porque as fêmeas atingiram a idade no ponto de inflexão com cerca de 323 dias, havendo uma desaceleração em seu crescimento. Ambos os sexos apresentaram mesmo crescimento relativo, porém nos machos o processo de desaceleração ocorreu em torno de dezoito dias após o ponto de inflexão das fêmeas, determinando as diferenças observadas no peso assintótico (Tabela 2).

A descrição do padrão de incremento do peso para machos e fêmeas, de acordo com o modelo de melhor ajuste, indicou que por aproximadamente 165 dias de vida machos e fêmeas possuíram crescimento semelhante, e após esta idade as fêmeas apresentaram crescimento lento, provavelmente devido à entrada em reprodução (Figura 2), redirecionando a energia utilizada do crescimento corporal para o crescimento das gônadas (BALDISSEROTTO, 2009). Em situações de cultivo de ciclo curto e menor peso ao abate, para a produção de processados, estes resultados indicam que o cultivo monossexo não precisaria ser praticado, reduzindo os custos da inversão sexual. Em avaliação da qualidade tecnológica de tilápias do Nilo enlatadas, Pizato et al. (2012) concluíram que o enlatado de tilápia fora do padrão (tamanho comercial inadequado) apresentou características físico-químicas, microbiológicas e sensoriais compatíveis com os produtos comerciais similares, confirmando a viabilidade do produto para o mercado de conservas, no qual estes resultados foram obtidos com tilápias de 5 a $10 \mathrm{~cm}$. No presente trabalho, os peixes apresentaram cerca de $15 \mathrm{~cm}$ de $\mathrm{CT}$ com aproximadamente 165 dias de idade (Figura 3).

Figura 2. Curvas de crescimento do peso em função da idade de machos (M) e fêmeas (F) de tilápias GIFT. Autora Aline Mayra da Silva Oliveira.

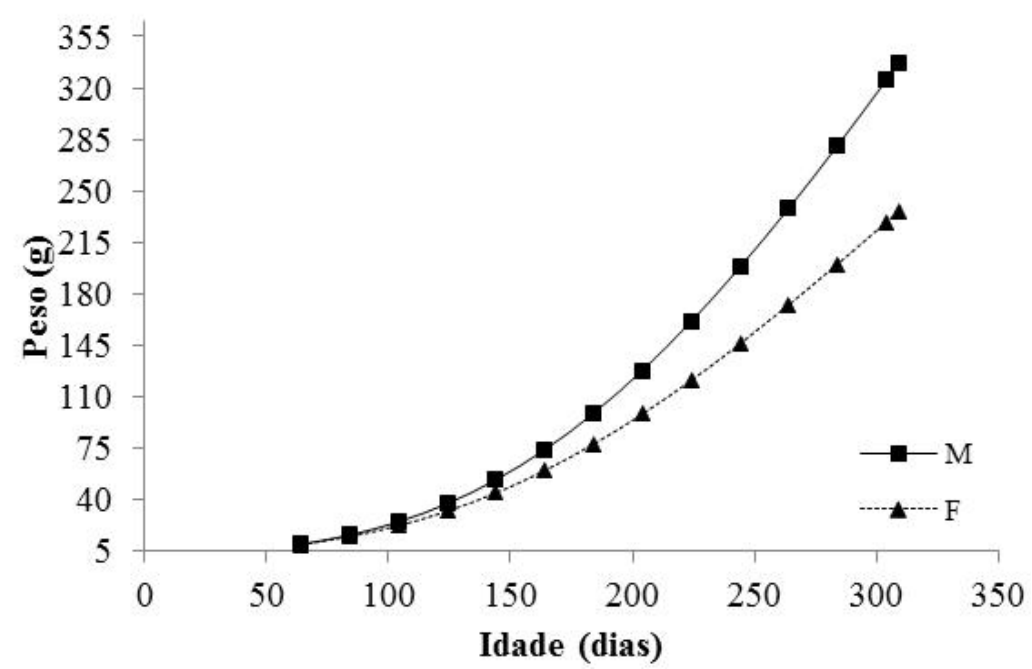

Fonte: Elaboração dos autores. 
Figura 3. Curvas de crescimento para comprimento total (CT), altura do corpo (AC), largura do corpo (LC) e tamanho da cabeça (TC) em função da idade de machos (M) e fêmeas (F) de tilápias GIFT. Autora Aline Mayra da Silva Oliveira
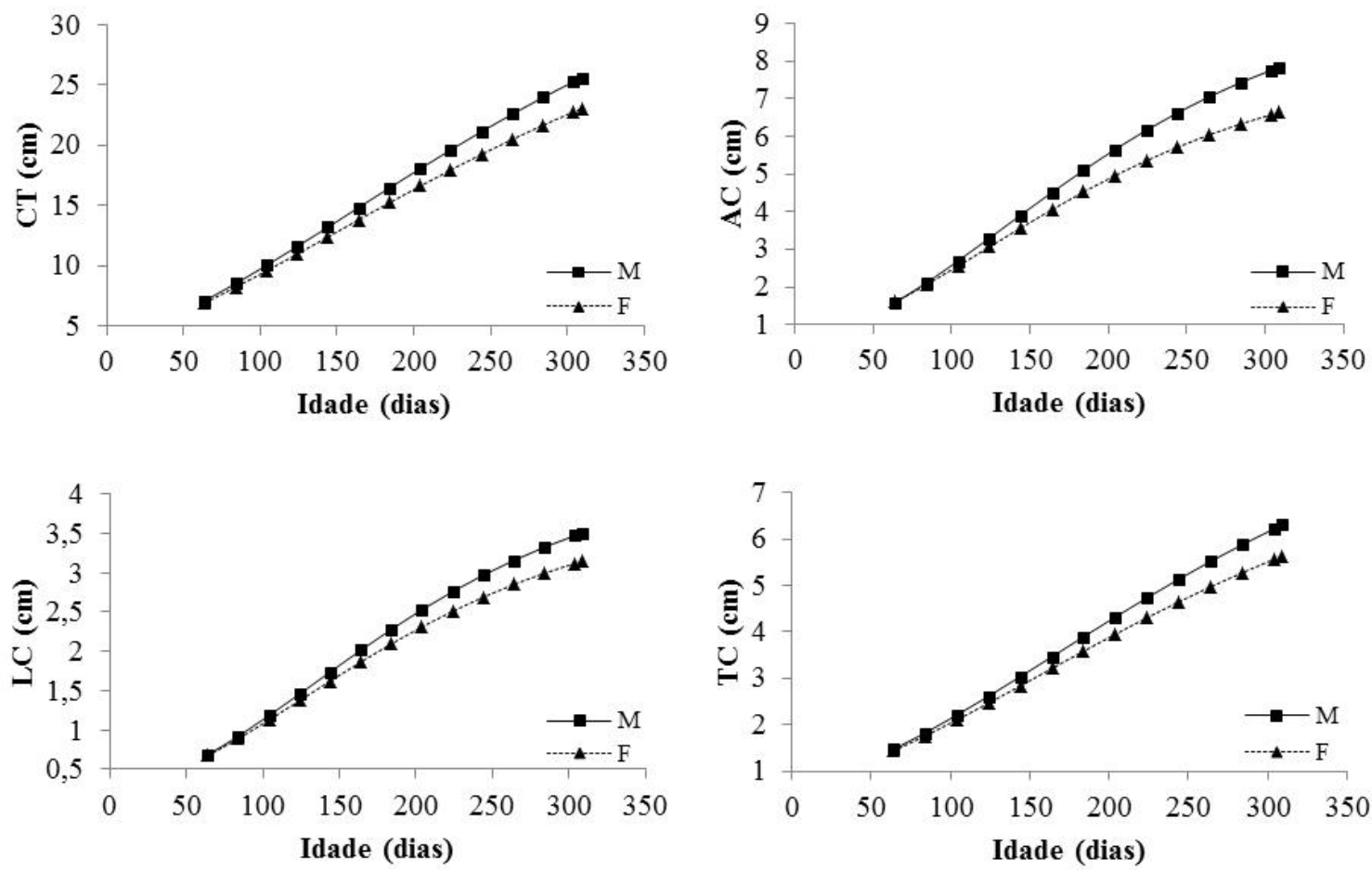

Fonte: Elaboração dos autores.

Diferenças nos pesos de machos e fêmeas em tilápias do Nilo desde idades mais jovens foram observadas por Rutten, Komen e Bovenhuis (2005). Estes mesmos autores ainda relataram que machos com aproximadamente 300 dias de vida apresentaram quase o dobro do peso corporal das fêmeas. No presente estudo o peso assintótico das fêmeas equivaleu a $62,5 \%$ do peso dos machos.

Devido às condições de manejo na fase de larvicultura e alevinagem, a idade dos peixes e seu desempenho foram diferentes das condições de cultivo comercial. Estas práticas impuseram restrição de espaço e pode ter interferido no crescimento inicial, o que possivelmente afetou o peso e a idade a maturidade estimados.

Quanto às características morfométricas CT, LC e TC foram verificados padrões de crescimento semelhantes ao descrito anteriormente para peso, no qual os valores assintóticos e a idade no ponto de inflexão para machos foram maiores que os valores encontrados para as fêmeas, confirmando a manutenção do crescimento em taxas elevadas por maior período de tempo nos machos (Tabela 2 e Figura 3).

A partir das estimativas do modelo 1 para $\mathrm{AC}$ observou-se que o crescimento relativo das fêmeas foi superior àquela estimada para os machos, porém o valor assintótico foi inferior para fêmeas devido às fêmeas atingirem mais rapidamente o ponto de inflexão (Tabela 2 e Figura 3). Segundo Fialho (1999) valores maiores de $b$ concentram o crescimento em torno do ponto de inflexão, aumentando a taxa de crescimento máximo, com isso seu crescimento inicial e final é mais lento. Por outro lado, valores menores de $b$ fazem com que o crescimento seja mais distribuído ao longo do tempo.

Em relação ao crescimento relativo das características $\mathrm{AC}$ e LC, estas foram maiores que 
o crescimento relativo das características $\mathrm{CT}$ e TC para ambos os sexos, o que indica crescimento mais rápido em altura e largura que em comprimento e tamanho da cabeça, atingindo seu crescimento máximo em menor período de tempo. Gomiero et al. (2009) ao avaliarem modelos de regressão não linear para descrever curvas de crescimento de piracanjuba (Brycon orbignyanus), obtiveram crescimento mais rápido em cabeça e largura que em comprimento padrão e altura do corpo em todos os modelos testados, incluindo o modelo Gompertz. $\mathrm{Na}$ análise de diversos modelos de regressão não linear para estimar a forma de crescimento de tilápias das linhagens Chitralada e Supreme, Santos et al. (2007b) encontraram, para o modelo Gompertz, valores semelhantes aos estimados no presente trabalho para AC e inferiores para LC nos machos.

Ao comparar o incremento do peso com as demais características, observou-se que os pontos de inflexão das características morfométricas variaram de 121,2 a 156,2 dias para fêmeas e 134,1 a 166,6 dias para machos, enquanto que o ponto de inflexão do peso foi de 340,4 e 322,9 dias para machos e fêmeas respectivamente, apontando padrões de incrementos na forma e massa diferentes, sendo determinada a forma dos peixes em idade menor. Isto indica que outras medidas corporais deveriam ter sido realizadas, pois percebeu-se deficiência na explicação do incremento no peso após o ponto de inflexão das características morfométricas. É provável que a largura ou altura em outros pontos do corpo do peixe tenha sofrido aumento acompanhando o incremento de peso, sugerindo aumento do volume corporal.

Segundo Contreras-Gusmán (1994) os peixes em geral possuem capacidade diferencial de acumulação de massa muscular em determinados pontos do corpo durante seu crescimento, o que caracteriza o seu formato e influencia os rendimentos cárneos. $\mathrm{O}$ mesmo autor afirma que as tilápias possuem a forma do corpo fusiforme com compressão lateral uniforme, possivelmente esta compressão está reduzindo com a seleção dos peixes. As medidas morfométricas segundo Bosworth, Libey e Notter (1998), contribuem para a descrição da forma do corpo do peixe e varia de acordo com as características de cada espécie, além de poderem influenciar o peso corporal e o rendimento do filé.

Verificou-se que a seleção dos peixes dentro do programa de melhoramento genético de tilápias do Nilo é realizada após o ponto de inflexão da característica $\mathrm{TC}$, ou seja, os peixes selecionados ainda aumentam o peso com a desaceleração do crescimento da cabeça, apresentando menor participação da cabeça no crescimento corporal dos peixes, ocorrendo aumento mais significativo de partes comestíveis.

Para as características avaliadas verificou-se que machos e fêmeas mantiveram seu crescimento com o avanço da idade (Figuras 2 e 3). Estes resultados estão de acordo com Weatherley e Gill (1987), que afirmam que os peixes podem ter crescimento contínuo se houver suprimento alimentar enquanto viverem. Todas as características morfométricas avaliadas no presente estudo aumentaram com o aumento do peso dos peixes, porém em velocidades diferentes. Em estudo do rendimento do processamento de linhagens melhoradas em função do peso, Santos et al. (2007a) concluíram que as linhagens Chitralada e Supreme possuíam crescimento das partes componentes do corpo proporcional ao aumento do peso corporal e que este aumento não alterou $\mathrm{o}$ rendimento desses constituintes.

\section{Conclusão}

O modelo de Gompertz é uma ferramenta útil na descrição do crescimento das tilápias melhoradas, auxiliando na avaliação das variáveis, por melhorar os pontos da curva de crescimento e selecionar as características desejáveis dentro do sistema de produção.

O padrão de crescimento das partes constituintes 
do corpo e do peso de machos e fêmeas de tilápias melhoradas é diferente, confirmando o dimorfismo sexual em tilápias do Nilo, apesar dos machos apresentarem mesmo crescimento relativo no ponto de inflexão que as fêmeas para a maioria das características avaliadas, com exceção de altura do corpo.

\section{Agradecimentos}

Ao Conselho Nacional de Desenvolvimento Científico e Tecnológico - CNPq pelo apoio financeiro.

\section{Referências}

BALDISSEROTTO, B. Fisiologia de peixes aplicada à piscicultura. 2. ed. Santa Maria: UFSM, 2009. 349 p.

BARICHELLO, F.; ALENCAR, M. M.; FREITAS, A. R.; BARBOSA, P. F. Curvas de crescimento em altura de fêmeas Nelore, Aberdeen Angus x Nelore, Canchim x Nelore e Simental x Nelore nascidas em duas estações do ano. In: REUNIÃO ANUAL DA SOCIEDADE BRASILEIRA DE ZOOTECNIA - SBZ, 46., 2009, Maringá. Anais... Maringá: Universidade Estadual de Maringá, 2009. CD-ROM.

BERG, R. T.; BUTTERFIELD, R. M. New concepts of cattle growth. Sydney: Sydney University Press, 1976. $240 \mathrm{p}$.

BORGES, A. I. C. Interface comparação de curvas de crescimento: aplicação informática para o auxilio na comparação de curvas de crescimento de populações de peixes. 2008. Dissertação (Mestrado em Ensino da Matemática) - Universidade do Porto, Porto.

BOSWORTH, B. G.; LIBEY, G. S.; NOTTER, D. R. Relationship among total weight, body shape, visceral components, and fillet traits in palmetto bass (Striped bass female Morone saxatilis x white bass male $M$. chrysops) and paradise bass (Striped bass female Morone saxatilis $\mathrm{x}$ yellow bass male M. mississippiensis). Journal of World Aquaculture Society, Baton Rouge, v. 29, n. 1, p. 40-50, mar. 1998.

CONTRERAS-GUZMÁN, E. S. Bioquímica de pescados e derivados. Jaboticabal: FUNEP, 1994. 409 p.

COSTA, A. C.; NETO REIS, R. V.; FREITAS, R. T. F.; FREATO, T. A.; LAGO, A. A.; SANTOS, V. B. Avaliação do crescimento de tilápias de diferentes linhagens através dos modelos não lineares. Archivos de Zootecnia, Córdoba, v. 58, p. 561-564, 2009. Suplemento 1.

FIALHO, F. B. Interpretação da curva de crescimento de Gompertz. Concórdia: Embrapa - CNPSA, 1999. 4 p. (Comunicado técnico, 237).

GAMITO, S. Growth models and their use in ecological modelling: an application to a fish population. Ecological Modelling, Amsterdam, v. 133, p. 83-94, nov. 1998.

GOMIERO, J. S. G.; FREITAS, R. T. F.; SANTOS, V. B.; SILVA, F. F.; RODRIGUES, P. B.; LOGATO, P. V. R. Curvas de crescimento morfométrico de piracanjuba (Brycon orbignyanus). Ciência e Agrotecnologia, Lavras, v. 33, n. 3, p. 882-889, maio/jun. 2009.

MAZZINI, A. R. A.; MUNIZ, J. A.; AQUINO, L. H.; SILVA, F. F. Análise da curva de crescimento de machos Hereford. Ciência e Agrotecnologia, Lavras, v. 27, n. 5, p. 1105-1112, set./out. 2003.

PIZATO, S.; KRAIESKI, J.; SARMENTO, C.; PRENTICE, C. Avaliação da qualidade tecnológica apresentada por tilápia do Nilo (Oreochromis niloticus) enlatada. Semina: Ciências Agrárias, Londrina, v. 33, n. 2, p. 667-674, abr. 2012.

REGAZZI, A. J.; SILVA, C. H. O. Teste para verificar a igualdade de parâmetros e a identidade de modelos de regressão não linear: dados no delineamento inteiramente casualizado. Revista de Matemática e Estatística, São Paulo, v. 22, n. 3, p. 33-45, 2004.

RUTTEN, M. J. M.; KOMEN, K.; BOVENHUIS, $\mathrm{H}$. Longitudinal genetic analysis of Nile tilapia (Oreochromis niloticus L.) body weight using a random regression model. Aquaculture, Amsterdam, v. 246, n. 1-4, p. 101-113, may 2005.

SANTOS, V. B.; FREITAS, R. T. F.; LOGATO, P. V. R.; FREATO, T. A.; ORFÃO, L. H.; MILIOTTI, C. L. Rendimento do processamento de linhagens de tilápias (Oreochromis niloticus) em função do peso corporal. Ciência e Agrotecnologia, Lavras, v. 31, n. 2, p. 554-562, mar./abr. 2007a.

SANTOS, V. B.; FREITAS, R. T. F.; SILVA, F. F.; FREATO, T. A. Avaliação de curvas de crescimento morfométrico de linhagens de tilápia do Nilo (Oreochromis niloticus). Ciência e Agrotecnologia, Lavras, v. 31, n. 5, p. 1486-1492, set./out. 2007b.

SAS Institute. SAS/STAT ${ }^{\circledR}$. User's guide: statistics SAS 9.1.3. Help and Documentation. Cary, NC: SAS Institute Inc., 2002, 2004.

WEATHERLEY, A. H.; GILL, H. S. The biology of fish growth. London: Academic Press, 1987. 443 p. 
